\title{
Readmission Following Hospitalization for Alcoholic Hepatitis: Costly or Cost-Effective?
}

\author{
James O'Beirne ${ }^{1,2}$ \\ Published online: 30 September 2019 \\ ○) Springer Science+Business Media, LLC, part of Springer Nature 2019
}

\begin{abstract}
Alcohol-related liver disease (ArLD), a rising cause of chronic liver disease in the USA, is now an increasingly common cause of death, especially in younger people, reflecting changing patterns of alcohol consumption [1]. Acute alcoholic hepatitis (AAH) is the most florid presentation of ArLD, comprised of coagulopathy, deep jaundice, and systemic inflammation. The early mortality of AAH is high at $\sim 25 \%$ and disappointingly has not improved over time, reflecting the lack of therapeutic progress in this area [2]. Indeed, the only successful treatments that improve survival beyond 30 days are prolonged and complete abstinence from alcohol and liver transplantation [3, 4]. In practice, abstinence is often difficult to achieve and liver transplantation for AAH is not widely available or applicable to the majority of patients. Therefore, the long-term prognosis of patients who survive an initial hospitalization with AAH is poor and is associated with significant healthcare resource requirements.
\end{abstract}

In recent years, the issue of readmission to hospital following an initial admission related to cirrhosis has emerged as a topic of some importance. Readmission appears to be frequent, costly, and associated with decreased survival. Readmission rates could be potentially modifiable and thus may represent a target for improvement in the quality of care and reduction in costs.

Several investigators have reported on readmission rates in cirrhosis and potential avoidance strategies. There are, however, limited data regarding readmissions for $\mathrm{AAH}$, and given that these patients are often younger with higher disease acuity than those with cirrhosis, an understanding of

James O'Beirne

James.obeirne@health.qld.gov.au

1 Department of Hepatology, Sunshine Coast University Hospital, Birtinya, QLD 4575, Australia

2 University of the Sunshine Coast, Sippy Downs, QLD, Australia readmission rates in this group and associated factors may be valuable.

In this issue of Digestive Disease and Sciences, Adejumo et al. [5] describe readmission rates, trends over time, and predictors of readmission in a retrospective cohort of patients from the National Readmissions Database (NRD), a representative sample from twenty states across the USA that provides data from ICD9 and procedure codes linked to unique patient identifiers that enable tracking of patient readmissions across multiple facilities within a state. Using this large database, the authors analyzed patients who survived their index admission and were discharged with a clinical diagnosis of AAH from 2010 to 2014. By analyzing patients admitted within the first 9 months of each year, they were able to examine readmissions at 30 days and at 90 days. In addition to measuring temporal changes in admission rates and costs, the authors attempted to identify demographic and clinical predictors of readmission.

Of the 21,572 patients identified with a discharge diagnosis of AAH, $22.8 \%$ were readmitted within 30 days and $36.6 \%$ were readmitted within 90 days. Factors associated with both 30- and 90-day readmissions were broadly similar: according to multivariate analysis, these predictors were female sex, ascites, leaving against medical advice, comorbidities as assessed by the Charlson-Deyo comorbidity index, and infections such as HCV and urinary tract infections. Referral to palliative care services reduced readmission rates at 30 and 90 days; younger age was associated with fewer readmissions at 90 days. Readmission rates and length-of-stay did not change across the study period though the number of patients discharged with a principal diagnosis of AAH increased from 3753 in 2010 to 5100 in 2014. This increase, associated with the general increase in healthcare costs over time, resulted in an increase of $\$ 56,000,000$ for 30 -day readmission costs and an increase of $\$ 115,000,000$ for 90-day readmissions over the study period.

How do these findings compare to what is already known about readmissions in cirrhosis generally? In a recent 
systematic review of 26 studies of cirrhosis, the 30-day readmission rate was $26 \%$ (range $10-50 \%$ ), whereas the 90 -day readmission rate was $21-71 \%$ [6]. Although the 30- and 90-day readmission rates in the current study are within this range (22.8\% and $36.6 \%$ ), one might imagine that they may have been higher given that patients with AAH are often of higher acuity than those with decompensated cirrhosis per se. Direct comparisons of the acuity of the AAH patients in the current study with previously published literature are unfortunately not possible as measures of disease severity such as MELD are not available within the NRD.

Of the predictors of readmission identified in previously published single-center and multicenter studies, the strongest are the MELD score, serum sodium, infection, ascites, hepatic encephalopathy (HE), and renal/metabolic problems [7]. Similar to the cirrhosis literature, Adejumo et al. found that although ascites and infections were associated with readmission, perhaps somewhat surprisingly HE did not appear to be significantly associated with readmission. One possible explanation for this finding is that HE was not coded for as a primary complication distinct from AAH within the NRD dataset. Such problems with coding and a lack of granularity are a feature of large administrative datasets, a point that is acknowledged by the authors and highlighted by others [8].

Several studies have examined different strategies aimed at a reduction in readmissions, a worthy goal in patients with AAH and cirrhosis. Strategies such as early follow-up either in the clinic or by telephone have met with varying success. Indeed, some interventions designed to reduce readmissions have paradoxically increased readmissions while reducing mortality [9]. Given these findings, the significance of the simple metric of 30- or 90-day readmission is unclear. The nature of complications of cirrhosis and their unpredictability such as variceal bleeding and infection means that some readmission is unavoidable. Moreover, as stated previously, some readmissions may reflect heightened vigilance toward complications and hence are a marker of high-quality care. Most studies have shown that readmissions are associated with $\mathrm{HE}$, ascites, and renal/metabolic derangements. Potentially, these are modifiable by redesigning discharge care to focus on education for caregivers and patients, easy access to ambulatory paracentesis, and early follow-up to detect complications of diuresis. Combining these aspects of care into a 'day hospital' model has shown efficacy in a quasiexperimental study, providing the rationale for a randomized controlled trial [10].

The findings of Adejumo et al. add to and expand the knowledge of the impact of readmissions in chronic liver disease. Reducing costs and improving quality where possible are important, especially when the overall prevalence of a disease is increasing. While interventions that are effective at reducing readmissions are needed, some readmissions are unavoidable and indeed may reflect high-value care. The principal priority must be at government level to reduce harmful alcohol intake by introduction of strategies such as minimum pricing and the provision of alcohol support agencies to prevent this cost burden in the first place.

\section{Acknowledgments None.}

Author's contribution JOB conceived the article and drafted the article. $\mathrm{JOB}$ is the sole guarantor of the article.

Funding No funding.

\section{Compliance with Ethical Standards}

Conflict of interest None declared.

\section{References}

1. Baki JA, Tapper EB. Contemporary epidemiology of cirrhosis. Curr Treat Options Gastroenterol. 2019;17:244-253. https://doi. org/10.1007/s11938-019-00228-3.

2. Hughes E, Hopkins LJ, Parker R. Survival from alcoholic hepatitis has not improved over time. PLOS ONE. 2018;13:e0192393. https ://doi.org/10.1371/journal.pone.0192393.

3. Altamirano J, López-Pelayo H, Michelena J, et al. Alcohol abstinence in patients surviving an episode of alcoholic hepatitis: prediction and impact on long-term survival. Hepatology. 2017;66:1842-1853. https://doi.org/10.1002/hep.29338.

4. Im GY, Cameron AM, Lucey MR. Liver transplantation for alcoholic hepatitis. J Hepatol. 2019;70:328-334. https://doi. org/10.1016/j.jhep.2018.11.007.

5. Adejumo AC, Cholankeril G, Iqbal U, et al. Readmission rates and associated outcomes for alcoholic hepatitis: a nationwide cohort study. Dig Dis Sci. (Epub ahead of print). https://doi.org/10.1007/ s10620-019-05759-4.

6. Orman ES, Ghabril M, Emmett TW, et al. Hospital readmissions in patients with cirrhosis: a systematic review. J Hosp Med. 2018;13:490-495. https://doi.org/10.12788/jhm.2967.

7. Bajaj JS, Reddy KR, Tandon P, et al. The 3-month readmission rate remains unacceptably high in a large North American cohort of patients with cirrhosis. Hepatology. 2016;64:200-208. https:// doi.org/10.1002/hep.28414.

8. Shaheen AA, Swain MG. Letter to Editor: using proper methods to identify patients with cirrhosis in administrative databases is crucial to correctly predict outcomes. Hepatology. 2019;69:23012302. https://doi.org/10.1002/hep.30343.

9. Kanwal F, Asch SM, Kramer JR, et al. Early outpatient followup and 30-day outcomes in patients hospitalized with cirrhosis. Hepatology. 2016;64:569-581. https://doi.org/10.1002/hep.28558

10. Morales BP, Planas R, Bartoli R, et al. HEPACONTROL. A program that reduces early readmissions, mortality at 60 days, and healthcare costs in decompensated cirrhosis. Dig Liver Dis. 2018;50:76-83. https://doi.org/10.1016/j.dld.2017.08.024.

Publisher's Note Springer Nature remains neutral with regard to jurisdictional claims in published maps and institutional affiliations. 